\title{
Usage of Microscopic Method to Ana- lyze Dispersion of Food Powders
}

\author{
Nickolay Pogozhikh, Igor Pavliuk, Andriy Foshchan , Zhanna Vorontsova
}

\section{KEY WORDS}

Microscopic method, dispersion of powders, screen analysis of powders, differentiated distribution function

\begin{abstract}
Affordable and balanced diet programmes gain popularity; hence major food manufacturers are interested in making inexpensive quality products. Very often, production of dietary foods requires nutritional powders with higher percentage of healthy ingridients. Therefore, the development of functional food powders is very perspective for the food industry. The variety of food powders allows their usage in production of different types of food. These food products are a source of biologically active substances such as vitamins, macro- and micronutrients. These elements are contained in an easily digestible form and in optimal proportions for the human body. However, a certain part of micronutrients is destroyed during the process of production of powders hence the partial loss of powders' nutritional value. Therefore, it is important to choose the optimal production of food powders that will allow to keep most important elements in place at an affordable price. For that reason, the main task of the production of food powders is to organize the most non-waste processing of raw resources and to keep intact food powders' constituent elements: vitamins, macro-and micronutrients, pectines for coloring, and other biologically active substances. Various methods of investigation of disperse systems provide a variety data for the control of food powders
\end{abstract}

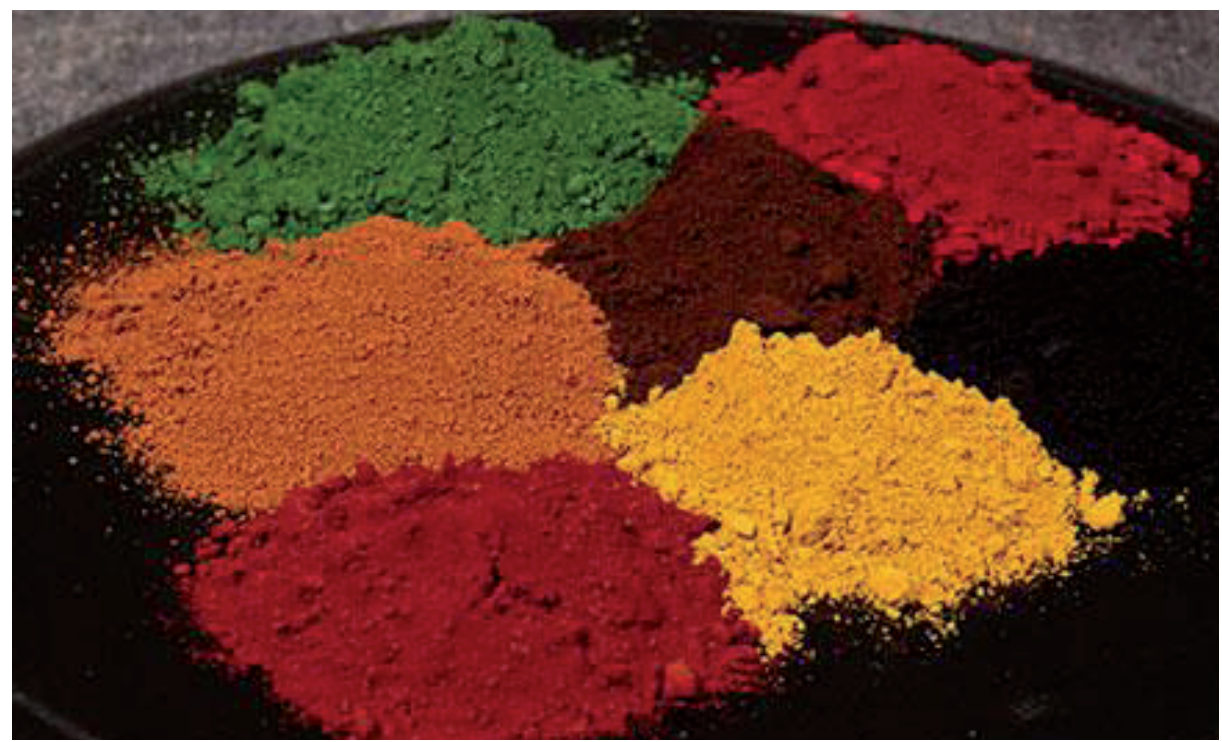

Fig. 1: Different types of food powders 


\section{INTRODUCTION}

Methods of dispersive analysis are widely used in analyzing of food raw material. There are several methods of dispersive analysis at the modern stage of development of science. Microscopic method differs by allowing not only to define geometrical dimensions of analyzed objects, but to see features of their form, texture and surface structure. The last fact is of high importance in cases, when powders are used further for preparation of reactive heterogeneous mixtures, which are used in food industry [1]. Microscopic method allows to measure particles 0,3-100 micrometers in dimension. The disadvantage of microscopic method is it's high laboriousness. Reliable results of determination of particles by microscopic method can be derived only by measuring of several hundreds of particles. Consequently duration of microscopic analysis could take several hours. This disadvantage is easily eliminated with help of modern computer technologies. Application of computer techniques allows not only to evaluate rapidly the average dimension of particles of powder, but also to define parameters of the distribution law describing dispersed composition of powdered food raw material in the best way [2].

\section{Calculation Methods}

To define dispersion of particles it is necessary to prepare samples in special way. Pattern of the powder is carefully mixed in full volume, with a needle the sample is carried on a slide with a water drop, which disperses. The particles of the sample are uniformly allocated on the drop and are covered with cover-glass. The cover-glass is carefully pressed to the sample to make monolayer of particles in the sample. After this microphotographies are taken $[1,3]$.

Prepared samples must be properly placed on the working surface of the microscope, images are adjusted to obtain clear boundaries between objects of research. These images are taken with the camera-recorder or photographic camera and recorded in electronic form. The microscopic bar is taken as well. For correct sample examination and averaging the data at least 5 fields from different parts of the sample are taken. The total number of analyzed objects must be at least $2000[2,4]$.

To describe dispersed composition of powders four equations are used: Goden-Andreev equation,
Rozin-Rammler equation, normal (ND) and lognormal distribution law (LND) [5]. Goden-Andreev equation derived under generalization of results of particle size analysis of powders disintegrated on various industrial equipment is following:

$$
D(\delta)=0,8\left(\frac{\delta}{A}\right)^{l}
$$

where: $D(\delta)$ - integral function of distribution, $\delta$ size of particles, $A$ - constant, parameter $l$ characterizes direction and rate of inflection of distribution curve: if $l=1$ curve changes to straight line; if $l<1$ curve $D(\delta)$ is convex; if $l>1$ curve $D(\delta)$ is concave.

By differentiation of it relative distribution function it comes out:

$\varphi(\delta)=0,8 \cdot l \cdot A^{-l} \cdot \delta^{l-1}$

In Rozin-Rammler equation [5] distribution curves according to screen analyses' data can be expressed by formula:

$$
D(\delta)=1-\exp \left[-\left(\frac{\delta}{B}\right)^{A}\right]
$$

where: A and B - constants, which are easily defined in logarithmic form of this equation according to research data.

As a result of previous equation density of mass distribution by diameters is defined by formula:

$\varphi(\delta)=\frac{A}{\delta}\left(\frac{\delta}{B}\right)^{A} \exp \left[-\left(\frac{\delta}{B}\right)^{A}\right]$

If $\mathrm{A}<1$ and $\delta \rightarrow 0$ distribution density $\varphi(\delta) \rightarrow$ $\infty$, though $D(\delta)$ if $\delta=0$ stays finite. Therefore if A $<1$ formula (4) doesn't give correct description of distribution of very small fractions. Normal distribution is normal Gaussian function, having the appearance as:

$D(\delta)=\frac{1}{\sigma \cdot \sqrt{2 \pi}} \int_{-\infty}^{\delta} \exp \left[-\frac{\left(\delta-\delta_{s 0}\right)^{2}}{2 \sigma^{2}}\right] d \delta$

where: $\delta$ so - median of distribution; $\sigma$ - meansquare of deviations of diameters from their mean value.

Differentiation of distribution function $D(\delta)$ by $\delta$ provides distribution density function:

$$
\varphi(\delta)=\frac{1}{\sigma \cdot \sqrt{2 \pi}} \exp \left[-\frac{\left(\delta-\delta_{s 0}\right)^{2}}{2 \sigma^{2}}\right]=\frac{1}{\sigma} F(t)
$$

where: $0<\delta<\infty$ 


$$
F(t)=\frac{1}{\sqrt{2 \pi}} \exp \left(-\frac{t^{2}}{2}\right)
$$

where: $t$ - normalized quantity normally distributed, which can be found in reference literature.

Log-normal distribution is obtained if the argument in normal Gaussian function is substituted not particles' diameter, but logarithm of diameter. LND function looks as:

$$
\begin{aligned}
& D(\delta)=\frac{1}{\ln (\sigma) \cdot \sqrt{2 \pi}} \cdot \\
& \int_{-\infty}^{\ln (\delta)} \exp \left[-\frac{\left(\ln (\delta)-\ln \left(\delta_{S 0}\right)\right)^{2}}{2 \ln ^{2}(\sigma)}\right] d \ln (\delta)
\end{aligned}
$$

where: $\delta$ so - distribution median; $\ln (\sigma)$ - meansquare mediation of logarithms of diameters from their mean value.

Differentiation of distribution function $D(\delta)$ by $\delta$ gives relative distribution function:

$$
\begin{aligned}
& \varphi(\delta)=\frac{1}{\sqrt{2 \pi} \cdot \delta \cdot \ln (\sigma)} \exp \left[-\frac{\left(\ln (\delta)-\ln \left(\delta_{s 0}\right)\right)^{2}}{2 \ln ^{2}(\sigma)}\right]= \\
& \frac{1}{\sqrt{2 \pi} \cdot \delta \cdot \ln (\sigma)} F(t)
\end{aligned}
$$

where: $0<\delta<\infty$. If distribution of particles' mass by dimensions follows log-normal law, then as well it will be followed by distributions of quantity and specific surface area of particles by dimensions [5].

\section{Practical Results and Discussion}

The model sample of food powder of various grinding was investigated. Shredding powder from food raw materials was obtained by mechanical milling in a mortar at a

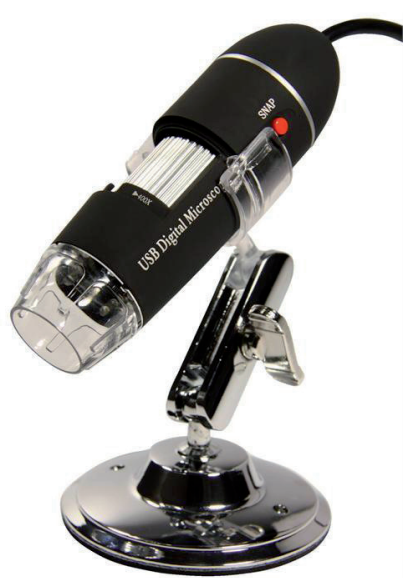

Fig. 2: USB Digital Microscope which was used in the experiment. constant speed during the prorated time. Obtained from food raw material powders of different dispersion were studied by means of USB Digital Microscope. After removing the microscopic bar the division value of microphotography was obtained. For each sample at least five fields from different angles of vision were shot. With photo-editing program PhotoM 1.21 linear dimensions of particles were defined. Results are presented in table.

Table 1: Number of particles of different dimension in food raw material powders with various dispersion during grinding.

\begin{tabular}{|l|l|l|l|l|}
\hline Intervals & $\mathbf{2}$ min. & $\mathbf{5}$ min. & $\mathbf{8} \mathbf{~ m i n .}$ & $\mathbf{1 2} \mathbf{~ m i n}$. \\
\hline 0.1 & 10 & 10 & 30 & 20 \\
\hline 1 & 70 & 140 & 80 & 60 \\
\hline 2 & 140 & 170 & 110 & 130 \\
\hline 3 & 170 & 250 & 140 & 160 \\
\hline 4 & 190 & 160 & 260 & 270 \\
\hline 5 & 160 & 130 & 210 & 190 \\
\hline 6 & 120 & 110 & 150 & 140 \\
\hline 7 & 60 & 50 & 60 & 60 \\
\hline 8 & 40 & 30 & 40 & 40 \\
\hline 9 & 20 & 20 & 20 & 20 \\
\hline
\end{tabular}

In this table columns (except the first) respond to powders of different dispersion, rows to the number of particles of different dimension. The highest dispersion has a powder in the fourth column, and the lowest - in the second, with it our calculations is started. First, the percentage of particles of different dimensions is found by the formula:

$z:=\frac{\operatorname{data}^{\langle 1\rangle}}{\sum_{i} \operatorname{data}_{i, 1}} \cdot 100 \%$

According to the formula [6] we divide amount of particles of defined dimension by total amount of particles and multiply by $100 \%$.

By formula:

$\sum Q=Q_{0}+Q_{1}+\ldots$

Integral function of distribution is build: 


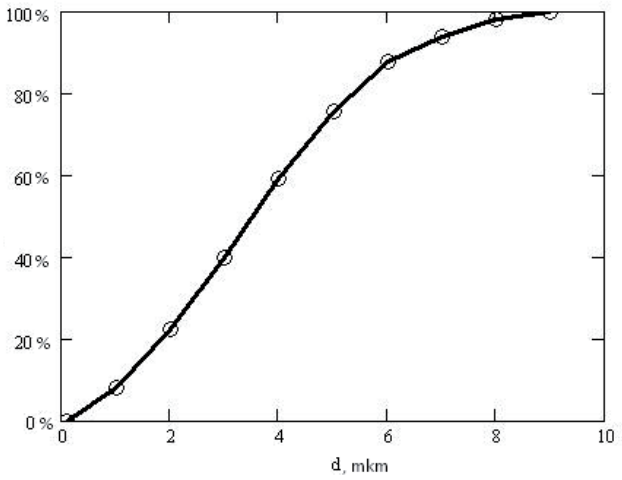

Fig. 3: Integral distribution function for powder $2 \mathrm{~min}$.

So the construction began with the smallest particles, by covering their percentage $\left(Q_{0}\right)$ on ordinate, then for the next fraction particles the ordinate was equal to the sum of percentage of particles prior fraction and the fraction of the larger radius and so on, working until the last ordinate (corresponding to the maximum diameter) will not make $100 \%$.

Then by following actions in Mathcad software environment:

$$
\begin{aligned}
& \begin{array}{l}
d:=\operatorname{data}^{\langle 0\rangle} \\
Y_{1}:=\underset{\ln (z)}{\longrightarrow}
\end{array} \quad F(x, a):=\left\{\begin{array}{l}
a_{1} \cdot x \cdot e^{a_{0}+a_{2} x} \\
a_{1} \cdot x \cdot e^{a_{0}+a_{2} x} \\
x \cdot e^{a_{0}+a_{2} x} \\
X_{1,0}:=1
\end{array}\right. \\
& \begin{array}{l}
X_{1}:=\frac{x^{3} \cdot e^{a_{0}+a_{2} x}}{\ln (d)} \\
X_{2}:=d
\end{array} \\
& \qquad a_{0}:=\left(X_{1}^{T} \cdot X_{1}\right)^{-1} \cdot X_{1}^{T} Y_{1} \\
& a_{1}:=\operatorname{genfit}\left(d, z, a_{0}, F\right)
\end{aligned}
$$

We build differentiating distribution function.

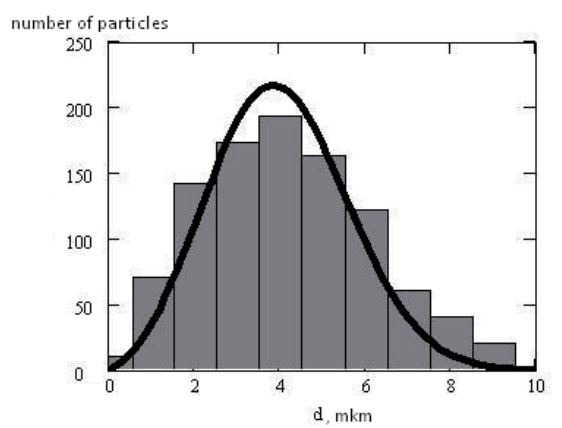

Fig. 4: Differentiating distribution function against the background of experimental discrete histogram pointing out amount of particles of different dimension (for powder $2 \mathrm{~min}$ ).
For approximation function of a type $a_{1} \cdot x \cdot e^{a_{0}+a_{2} x}$ has been used. As it could be seen it sharply estimates experimental data and, that's uppermost, precisely coincides with experimental maximum, which allows to define by this function the most likely dimension of particles for given sample [7]. The work with data for other samples is analogous.

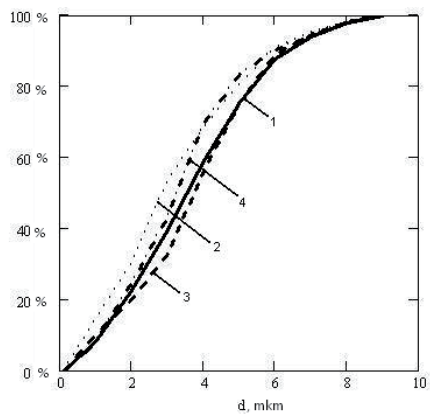

Fig. 5: Integral distribution functions for four modeling powders of different dispersion.

1-curve for 2-min.powder (solid line)

2-curve for 5-min. powder (dot line)

3-curve for 8-min. powder (dashed line)

4-curve for 12-min. powder (dot-dash)

To define average dimension of powders from food raw material we figure all differentiating distribution function at one coordinate system.

\section{Summary}

After analyzing different methods for determining the dispersion of powders microscopic method was selected for the study of food raw material powders, which allows to obtain much information about the features of shape, texture and surface structure of observed samples of food raw materials. The study of modeling samples of food raw materials of various grinding allowed to develop a method of calculating the dispersion of food powders. In the analysis obtained during the experiment of dispersion curves selection of the most rational approximation curves for powders of different dispersion was carried out. In our view, a comparative analysis of two pairs of food powders with significantly different dispersion was appropriate.

This work is performed according to the state budget theme № 2-11 FB "Research of condition and the structure of water in food substance by NMR and EPR spectroscopy". 


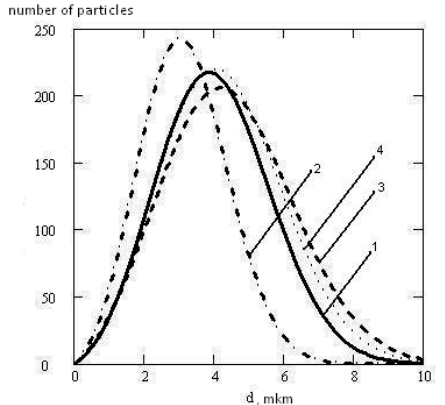

Fig. 6: Differentiating distribution function for four modeling powders of different dispersion.

1-curve for 2-min.powder (solid line)

2-curve for 5-min. powder (dot-dash)

3-curve for 8-min. powder (dashed line)

4-curve for 12-min. powder (dot line)

\section{References}

[1] Fayed, M.; Otten, L.: Handbook of powder sience. Technolog, Carman-Hall, New York, 1997

[2] Квеско, Н.: Оценка точности и области применения некоторых методов анализа дисперсности порошков, Сибирский физико-технический журнал СО АН СССР, Новосибирск, 1992

[3] Петрунин, В.: Ультрадисперсные (нано-) материалы и нанотехно-Логии, Инженерная физика, 2001

[4] Kunze, K.; Wright, S.: Textures and Microstructures. Vol.20 (1993), pp.41-54

[5] Коузов, П.: Основы анализа дисперсного состава промышленных пылей и измельченных материалов, Химия, Ленинград, 1987

[6] Кирьянов, Д.: Вычислительная физика, Полибук Мультимедиа, Москва, 2006

[7] Максфилд, Б.: Mathcad в инженерных расчетах, КОРОНАВек, Санкт Петербург, 2010

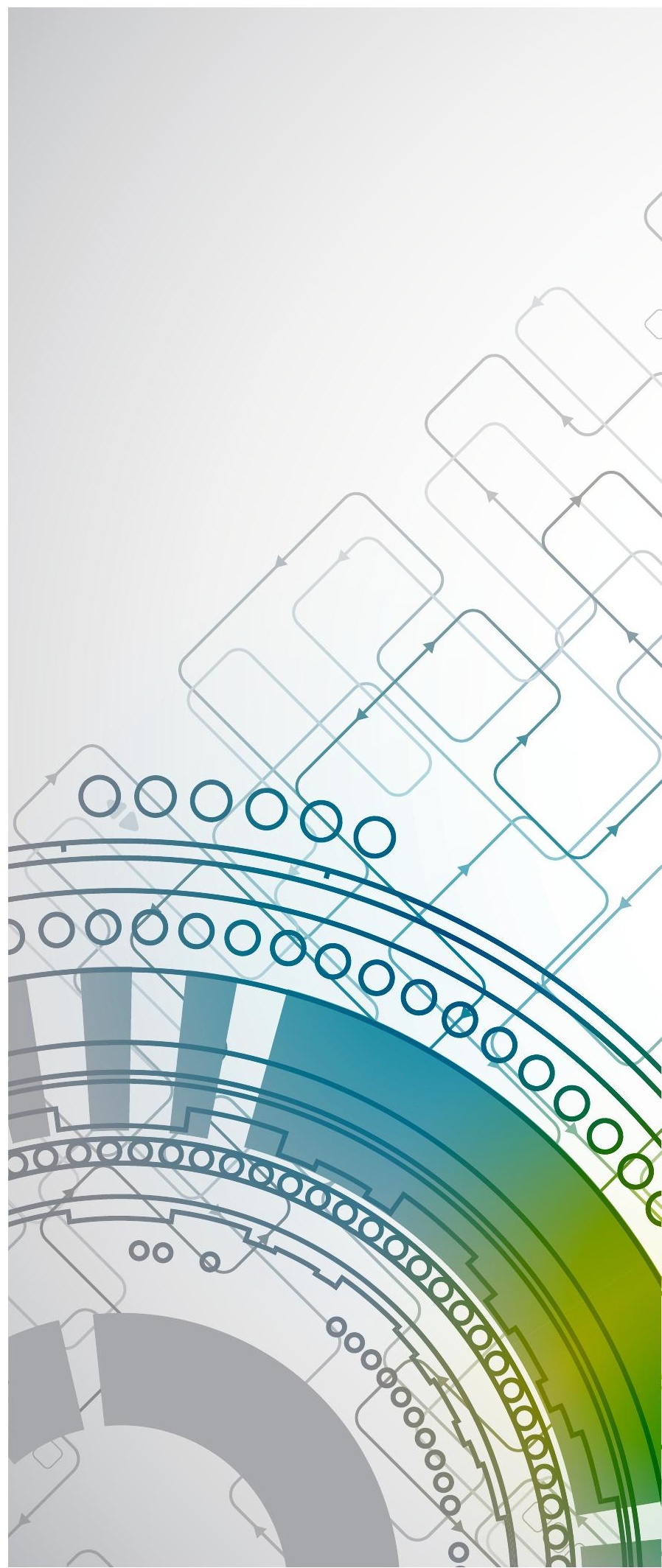




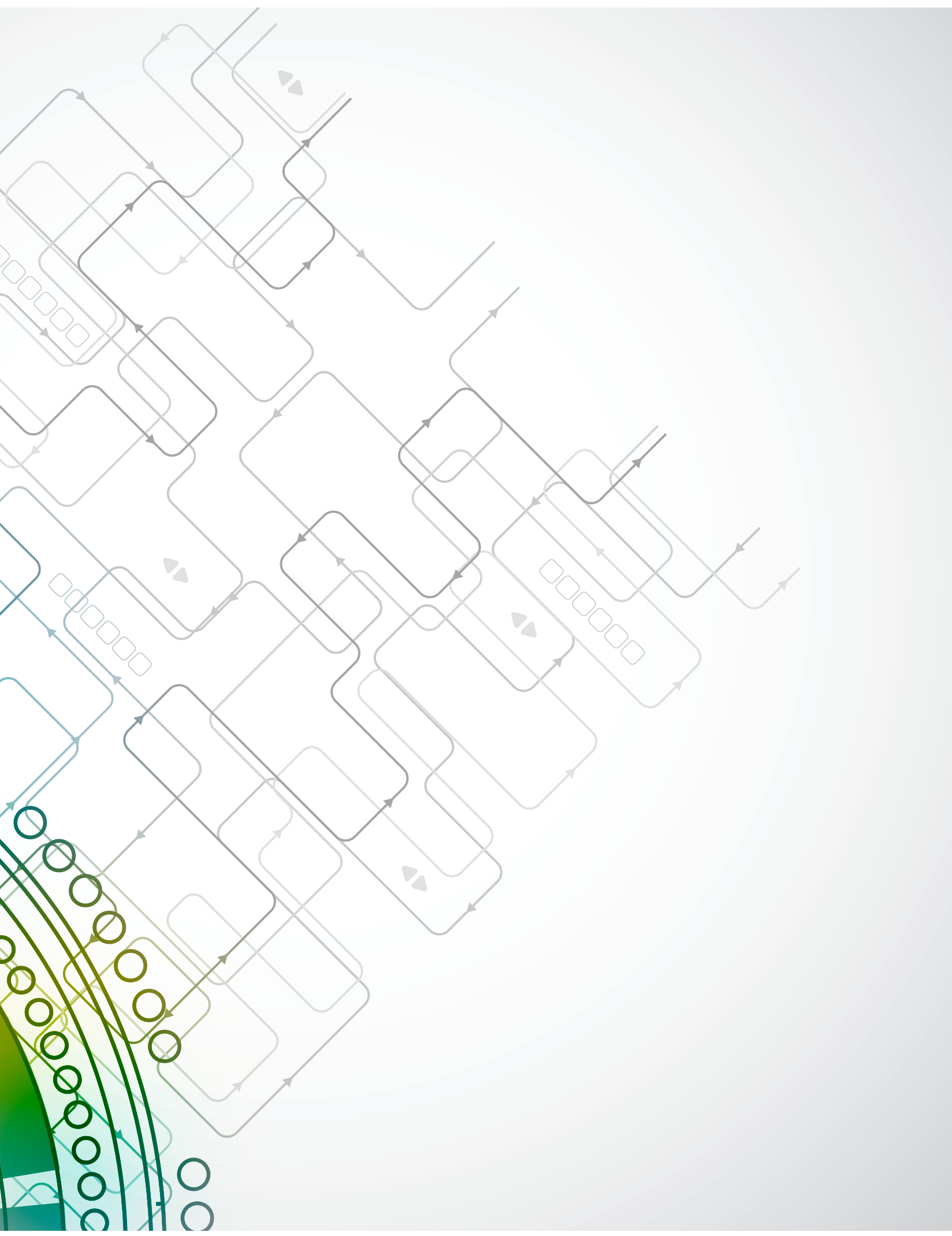

